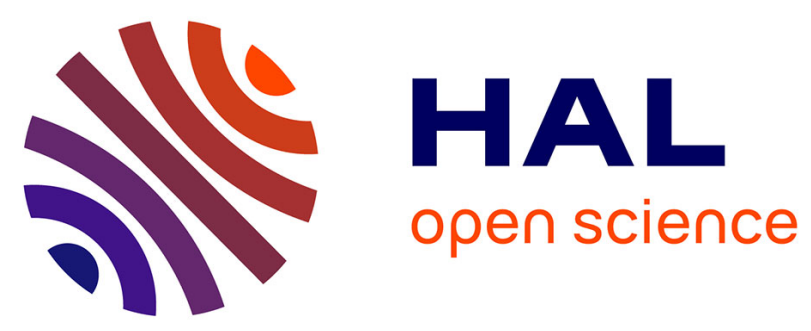

\title{
SAISIR LES PRATIQUES SOCIALES DU POINT DE VUE DE LEUR ORGANISATION. REVISITER LE CONCEPT DE MÉDIATION
}

Marcela Patrascu

\section{> To cite this version:}

Marcela Patrascu. SAISIR LES PRATIQUES SOCIALES DU POINT DE VUE DE LEUR ORGANISATION. REVISITER LE CONCEPT DE MÉDIATION. Les Enjeux de l'information et de la communication, 2010, Dossier 2 "La/les médiation(s) en SIC", 2/2010, pp.58-70. hal-02332108

\section{HAL Id: hal-02332108 \\ https://hal.science/hal-02332108}

Submitted on 24 Oct 2019

HAL is a multi-disciplinary open access archive for the deposit and dissemination of scientific research documents, whether they are published or not. The documents may come from teaching and research institutions in France or abroad, or from public or private research centers.
L'archive ouverte pluridisciplinaire HAL, est destinée au dépôt et à la diffusion de documents scientifiques de niveau recherche, publiés ou non, émanant des établissements d'enseignement et de recherche français ou étrangers, des laboratoires publics ou privés. 


\title{
SAISIR LES PRATIQUES SOCIALES DU POINT DE VUE DE LEUR ORGANISATION. REVISITER LE CONCEPT DE MÉDIATION
}

\author{
Marcela Patrascu
}

GRESEC | « Les Enjeux de l'information et de la communication »

2010/2 Dossier 2010 | pages 58 à 70

Article disponible en ligne à l'adresse :

https://www.cairn.inforevue-les-enjeux-de-l-information-et-de-lacommunication-2010-2-page-58.htm

Distribution électronique Cairn.info pour GRESEC.

(C) GRESEC. Tous droits réservés pour tous pays.

La reproduction ou représentation de cet article, notamment par photocopie, n'est autorisée que dans les limites des conditions générales d'utilisation du site ou, le cas échéant, des conditions générales de la licence souscrite par votre établissement. Toute autre reproduction ou représentation, en tout ou partie, sous quelque forme et de quelque manière que ce soit, est interdite sauf accord préalable et écrit de l'éditeur, en dehors des cas prévus par la législation en vigueur en France. Il est précisé que son stockage dans une base de données est également interdit. 


\section{Saisir les pratiques sociales du point de vue de leur organisation. Revisiter le concept de médiation}

Article inédit. Mis en ligne le 21 décembre 2010.

\section{Marcela Patrascu}

Marcela Patrascu est doctorante/monitrice en Sciences de l'Information et de la communication (sous la direction de Christian Le Moënne), à l'Université Rennes 2, au sein du laboratoire PREFics. Elle réalise sa thèse avec le soutien financier de la Région Bretagne.

Plan

Introduction

Usage, pratiques : quelles problématiques derrière une terminologie ambiguë ?

La dimension organisationnelle des pratiques sociales comme problématique heuristique dans les approches des Tic

La médiation : un concept fédérateur

Conclusion

Références bibliographiques

\section{Résumé}

L'objectif de cet article est de revisiter les concepts de "pratique », " organisation » et "médiation ». Il s'agit de rendre compte de leurs complémentarités mais également des tensions qui surgissent lorsqu'elles sont «pensées » ensemble. La notion de pratique, plus riche théoriquement que la notion d'usage (B. Miège, 2007), pose dans une approche communicationnelle la question des objets techniques en tant que médiateurs. La médiation est alors vue comme articulation entre l'individuel et le collectif. Car les gens organisent sans cesse leurs pratiques et leurs actions dans l'espace public en mettant en commun un ensemble de normes et codes, en se rendant mutuellement intelligibles. (L. Quéré, 1998). Saisir la dimension organisationnelle des pratiques sociales (Ch. Le Moënne, 2004) revient donc à saisir les tensions entre des normes et formes sociales héritées et celles émergentes. Selon F. Bernard, (2000) c'est justement la notion de médiation qui permet de dépasser la logique binaire qui oppose sujet et objet, individu et organisation, technique et social et de penser la triade « individu-technique-organisation ».

\section{Abstract}

This paper discusses the concepts of practice, organisation and mediation. It's aim is to revisit their complementarities but also the tensions that arise when this concepts are "thoughts" together. The concept of practice, theoretically richer that the concept of use (B. Miege, 2007), questions in an communicative approach the technical objets as mediators. Mediation is then seen as an articulation between the individual and the collective. Indeed, people always organize their practices and actions in public space by sharing a set of rules and codes (L. Quéré, 1998). For Ch. Le Moënne (2004) the organizational dimension of social practices is maded of permanent tensions between the social norm inherited from the past and new social norms emerging. The concept of mediation overcomes the binary logic that opposes subject and object, individual and organization, technology and society. This concept is a tool that allows us to think the triad "individual-technology-organization" (F. Bernard, 2000).

\section{Resumen}

Este artículo discute los conceptos de la práctica, la organización y la mediación. Su objetivo es volver a examinar las complementariedades y las tensiones que surgen cuando 
estos conceptos son pensados juntos. Punto de vista teórico, el concepto de la práctica es más rico que el concepto de uso (B. Miège, 2007) : los objetos técnicos son considerados como mediadores y la mediación es vista como una articulación entre el individual y el colectivo. La gente siempre organiza sus prácticas y acciones en el espacio público a través de las normas y códigos sociales que tienen en común (L. Quéré, 1998). Para Christian Le Moënne (2004), la dimensión organizativa de las prácticas sociales implica una tensión permanente entre las normas sociales heredadas del pasado y las normas sociales nuevas nuevas. Según F. Bernard, (2000) es justamente la noción de mediación que permite sobrepasar la lógica binaria que opone sujeto y objeto, individuo y organización, técnico y social y de pensar en la tríada "individuo-tecnología-organización."

\section{INTRODUCTION}

Les notions de pratique, d'organisation et de médiation sont souvent mobilisées dans les travaux de recherche en sciences de l'information et de la communication. La notion de pratique, plus large et plus riche théoriquement que la notion d'usage (Miège, 2007), pose dans une approche communicationnelle la question des objets médiateurs et de la médiation, vue comme articulation entre l'individuel et le collectif. Car les gens organisent sans cesse leurs pratiques et leurs actions dans l'espace public en mettant en commun un ensemble de normes et codes, en se rendant mutuellement intelligibles (Quéré, 1998). Saisir la dimension organisationnelle des pratiques sociales (Le Moënne, 2004) revient donc à saisir les tensions entre des normes et formes sociales héritées et celles émergentes telle qu'elles se donnent à voir en situation d'interaction multimodale entre usager/objet/environnement. Selon Françoise Bernard (2000, p. 36), c'est justement la notion de médiation qui permet de dépasser la logique binaire qui oppose sujet et objet, individu et organisation, technique et social et de penser la triade "individu-techniqueorganisation ». L'objectif de cet article est de revisiter ces notions et de rendre compte de leurs complémentarités, mais également des tensions qui surgissent lorsqu'elles sont «pensées » ensemble.

Notre argumentation s'efforcera d'apporter plus de précisions d'ordre terminologique et conceptuel sur les notions qui sont au cœur de notre questionnement: organisation, pratique et médiation.

Nous poursuivrons cette réflexion en examinant comment les frontières entre ces trois notions peuvent être dépassées à travers une approche communicationnelle des organisations au sens large qui reposerait sur une prise en compte des médiations (techniques, sociales et symboliques) et des objets (vus comme médiateurs) dans l'action. En poursuivant une orientation donnée par Jean Caune nous allons montrer que la notion de médiation reste riche du point de vue heuristique : elle permet toujours de faire avancer les réflexions autour d'un renouvèlement des approches des Tic et de l'innovation dans le champ des sciences de l'information de la communication. Notion du sens commun, transformée en concept, la médiation permet de "penser ensemble " les notions de pratique et d'organisation aussi bien dans un axe horizontal en permettant de saisir la dimension organisationnelle des pratiques individuelles et collectives, mais également dans un axe vertical, celui qui oriente l'appréhension de l'innovation inscrite dans une temporalité longue. 


\section{USAGES, PRATIQUES : QUELLES PROBLEMATIQUES DERRIERE UNE TERMINOLOGIE AMBIGUË ?}

Plusieurs chercheurs (Y. Jeanneret, B. Miège, C. le Moënne) pointent, voire même désapprouvent, le tournant que les approches des technologies de l'information et de la communication ont pris dans notre discipline. Avec le développement des multiples technologies nomades, les études dites d'usages des Tic gagnent du terrain. Bernard Miège considère que la sociologie des usages semble être devenue "une voie privilégiée dans l'approche des TIC » (Miège, 2007). Yves Jeanneret « en tant que chercheur formé dans les années soixante-dix ", se déclare «impressionné par la façon dont (la notion d'usage) a remplacé celle de pratique ». Il n'est d'ailleurs pas rare que des suspicions sur leur caractère "pro-innovateur ", « opérationnel ou opérationnable " (Miège, 2007) soient formulées. Il convient de se demander de quelle façon ces études peuvent être utilisées sans tenir compte de leur mode de construction et de ceux qui les commandent ?

Dans un souci pour l'emploi d'une terminologie adéquate, d'autres termes du même champ sémantique auraient pu être associés à cette approche comparative, comme par exemple, les notions d'utilisation et d'appropriation. Dans un schéma, tout à fait incomplet et manquant de nuances, nous avons esquissé une comparaison entre ces notions, leurs acceptions et leurs «instrumentalisations ». Nous avons fait l'impasse sur une grande partie des travaux portant sur une appréhension complexe des dispositifs socio-techniques, notamment la socio-politique des usages et la sociologie de l'innovation.

\begin{tabular}{|c|c|c|c|c|}
\hline notion & utilisation & usage & appropriation & pratique \\
\hline définition & $\begin{array}{l}\text { «Emploi } \\
\text { d'une } \\
\text { technique» }\end{array}$ & $\begin{array}{l}\text { «face à face } \\
\text { restreint entre } \\
\text { l'usager et le } \\
\text { dispositif } \\
\text { technique » }\end{array}$ & $\begin{array}{l}\text { > vb.lat. appropriare, } \\
\text { " faire sien" }\end{array}$ & $\begin{array}{l}\text { Notion plus large } \\
\text { qui englobe } \\
\text { l'usage, les } \\
\text { comportements, les } \\
\text { présentations, les } \\
\text { imaginaires. }\end{array}$ \\
\hline $\begin{array}{l}\text { Notions } \\
\text { complé- } \\
\text { mentaires }\end{array}$ & $\begin{array}{l}\text { Contrainte } \\
\text { technique }\end{array}$ & $\begin{array}{l}\text { - braconnages, } \\
\text { - réinventions, } \\
\text { - discours des } \\
\text { usagers }\end{array}$ & $\begin{array}{l}\text { - adaptation } \\
\text { - appropriation } \\
\text { individuelle/appropri } \\
\text { ation collective }\end{array}$ & $\begin{array}{l}\text { Médiations } \\
\text { techniques, sociales } \\
\text { et symboliques. } \\
\text { Dimension socio- } \\
\text { politique, stratégies } \\
\text { des macro-acteurs. }\end{array}$ \\
\hline $\begin{array}{l}\text { Applica- } \\
\text { tions, } \\
\text { ancrage } \\
\text { " discipli- } \\
\text { naire" }\end{array}$ & Ergonomie & $\begin{array}{l}\text { Uses } \mathcal{E}^{2} \text { Gratifications, } \\
\text { la Sociologie des } \\
\text { usages, SIC }\end{array}$ & études d'anticipation & $\begin{array}{l}\text { SIC, études des } \\
\text { médiations. }\end{array}$ \\
\hline $\begin{array}{l}\text { Dérives } \\
\text { possibles } \\
\text { et } \\
\text { difficultés }\end{array}$ & $\begin{array}{l}\text { Déterminisme } \\
\text { technique }\end{array}$ & $\begin{array}{l}\text { Focalisation } \\
\text { exclusivement au } \\
\text { niveau micro } \\
\text { (discours des } \\
\text { usagers) }\end{array}$ & Déterminisme social & $\begin{array}{l}\text { Difficulté } \\
\text { méthodologique } \\
\text { pour réconcilier le } \\
\text { niveau micro de } \\
\text { l'analyse avec le } \\
\text { niveau macro. }\end{array}$ \\
\hline
\end{tabular}


Revenons sur les différences et les complémentarités entre les notions d'usage et de pratique qui dans leur polysémie, sont souvent employées l'une pour l'autre.

La notion d'usage est plus restrictive par rapport à la notion de pratique. Elle s'inscrit dans le cadre d'un questionnement sociologique et prend en compte un face-à-face restreint entre le dispositif et l'usager. (Jouët, 1993, p. 371). Le terme est apparu dans la sociologie des médias avec le courant fonctionnaliste des "Uses and gratifications » dans les travaux américains des années 1960 et 1970 . Les porteurs de ce courant cherchaient à prendre distance avec le paradigme dominant de l'époque - le déterminisme technique. En France, le livre de Michel de Certeau L'invention du quotidien paru en 1980, influencera les études pourtant sur les usages des Tic. Dans le rapport usager/dispositif technique, les chercheurs vont favoriser l'usager. On s'intéresse à ce que les usagers font avec les objets techniques, à leurs «arts de faire », à leur capacité à détourner les usages prescrits, et surtout à leur discours. Pour Yves Jeanneret «sur le plan visuel, une étude d'usage suggère un individu face à un dispositif dont il parvient ou ne parvient pas à utiliser les fonctionnalités ».

Ainsi, à l'image d'une technologie structurante dont les caractéristiques déterminent les usages sociaux, s'est substituée la conception d'une technologie "molle ", objet d'une construction sociale aussi bien lors de sa phase de production (la sociologie de la traduction) que celle de sa diffusion. Selon Méadel et Proulx, parler de la notion d'usage signifie déjà "s'inscrire dans une problématique sociologique traditionnelle de l'usage non comme face-à-face d'un individu et d'un objet, mais plutôt braquer le projecteur vers l'individu " (Méadel, Proulx, 1993, p. 3). Mais en «braquant le projecteur» vers l'usager, on ne regarde que la moitié des phénomènes, qu'un côté des choses. Les méthodologies sont souvent logocentriques, basées sur des entretiens approfondis avec les usagers, le plus souvent semidirectifs et enregistrés. Bien avant le développement croissant des systèmes mobiles, Jacques Perriault (1989) remarquait la difficulté d'observer les usages et pointait les limites de l'utilisation exclusive des entretiens : "l'usage est très difficile à observer. (...) La personne observée n'a souvent qu'une Avant conscience partielle de ce qu'elle est en train de faire. L'entretien ne suffit donc pas. Il faut regarder, et pour comprendre ce qu'on voit, savoir pratiquer soi-même ». Avoir seulement le point de vue des usagers met d'entrée de jeu les acteurs au premier plan et les objets techniques au deuxième. De plus, la question du rapport entre le "dire » et le "faire" reste très problématique dans les méthodologies basées exclusivement sur entretiens approfondis. Elles ne réussissent pas à surmonter un défi épistémologique et méthodologique : comment dépasser le simple niveau de déclaration des usagers sur leur propre pratique lors des entretiens ? (Proulx, 2005).

La notion de pratique est au contraire plus riche et plus large. Josiane Jouët fait une différenciation assez claire entre les deux notions : «l'usage est [...] plus restrictif et renvoie à la simple utilisation tandis que la pratique est une notion plus élaborée qui recouvre non seulement l'emploi des techniques (l'usage), mais les comportements, les attitudes et les représentations des individus qui se rapportent directement ou indirectement à l'outil » (Jouët, 1993, p. 371). Pour Bernard Miège, les pratiques : " ne se limitent pas à l'usage d'une Tic ou à la fréquentation de tel spectacle ou de telle activité (...) les pratiques font référence à toute une série de représentations sociales - symboliques, y compris à des schémas relevant de l'imaginaire, qui ne permettent pas d'identifier clairement et immédiatement les significations dont elles sont porteuses» (Miège, 2007, p. 173).

Les chercheurs qui militent pour un retour des approches des Tic à la notion de pratique (B. Miège, J. Jouet, Y. Jeanneret, Ch. Le Moënne) encouragent le dépassement des approches méthodologiques « logocentriques » qui prennent en considération uniquement les productions langagières accompagnant l'action. Ils incitent les chercheurs en SIC à imaginer des méthodologies permettant de dépasser le niveau micro- des discours des 
usagers (S. Proulx, 2005, B. Miège, 2007). Ainsi, à l'instar de Louis Quéré, nous considérons qu'il est nécessaire que les approches des Tic en SIC dépassent les méthodologies basées sur les discours des usagers. La question sous-jacente est de savoir si "les agents savent ce qu'ils font, orientent consciemment leurs actions et leurs paroles, agissent en fonction d'un vouloir-faire et d'un vouloir-dire » (Quéré, 1990, p. 85).

Dans notre projet de recherche en cours, nous nous intéressons aux pratiques de visionnage de contenus audio-visuels en condition de mobilité et plus précisément aux usages de la télévision sur le téléphone portable. Le dispositif méthodologique mis en place vise une appréhension multimodale de l'interaction entre l'usager, l'objet et l'environnement. Il s'agit donc d'une approche qui porte à la fois sur les discours, les conduites et les maniements des artefacts et qui se base sur des enregistrements audiovisuels, de l'observation participante et des entretiens avec les usagers: entretiens d'explicitation (Vermersch, 1994) et entretiens d'auto-confrontation (Brassac, 2005). Le dispositif retenu articule deux prises de vue et associe une paire de lunettes-caméra (caméra subjective-portée par les usagers) et une autre caméra, qui filme l'interaction avec le système d'un point de vue plus large. Notre volonté de s'inscrire dans une démarche comparative exigeait des contextes quasi identiques pour les deux terrains d'étude. Les captures d'image et les observations ont été menées dans des lieux publics tels les transports en commun, les places publiques ou les restaurants afin de pouvoir observer les interactions complexes et les mises en forme des pratiques à la fois langagières, objectales et de conduite. Plusieurs captures vidéo ont été réalisées (une cinquantaine) avec une durée totale de plus de dix heures de film. Notre analyse a essayé des prendre en compte "des registres d'actions différents, selon que la prise en compte d'une autre ou d'autres personnes, pèse plus ou moins sur le déroulement de l'action et le jugement sur son accomplissement»(Thévenot, 1990). A travers nos choix méthodologiques, nous avons voulu déplacer l'attention consacrée aux descriptions des pratiques par les usagers, pour la porter sur l'analyse des formes et normes émergentes qui se manifestent au niveau langagier, gestuel, objectal.

En effet, à la base de cette distinction entre la notion d'usage et la notion de pratique se retrouvent deux positions épistémologiques contraires liées à l'émergence du sens des objets sociaux. Le sens des objets sociaux émerge selon nous dans le domaine du concret et du sensible, et non pas dans celui de la représentation et du discours rationnel.

Dans une telle perspective, ce n'est plus le discours, mais l'organisation des pratiques qui devrait constituer l'objet de l'analyse.

\section{LA DIMENSION ORGANISATIONNELLE COMME PROBLEMATIQUE HEURISTIQUE DANS LES APPROCHES DES TIC}

Mais qu'est-ce qu'une organisation pour les sciences de l'information de la communication ? Cette question suscite encore des débats dans notre discipline et le lien avec la notion de communication est sans cesse reformulé. Si l'on regarde l'évolution du champ de la communication des organisations, on observe bien que derrière les multiplies terminologies (voire hésitation lexicale) se cache non seulement une volonté de légitimité scientifique, mais aussi l'intention de «mieux» rendre compte des définitions que l'on donne à l'organisation et des manières d'appréhender la relation organisationcommunication. Ainsi, les chercheurs hésitent entre le singulier «communication organisationnelle» (N. d'Almeida et Y. Andonova, 2006 ; Ch. Le Moenne, 2000) et le pluriel «communications organisationnelles» (Delcambre, 2000) dans une volonté de dissociation du champ professionnel de la «communication d'entreprise». A l'heure 
actuelle, deux syntagmes semblent s'imposer par leur récurrence, mais aussi leurs apports théoriques : «la communication des organisations » (Colloque Org\&Co, Rennes, 2009) et ACO pour «approches communicationnelles des organisations" (Bouillon, Bourdin, Loneux, 2007, 2008).

Mais derrière ces terminologies, ce sont de différentes manières d'appréhender l'organisation qui se confrontent. Chaque syntagme choisi semble être porteur d'une conception différente de l'organisation. Par exemple derrière la terminologie "communication dans les organisations", on retrouve la vision d'une organisationcontenant, qui «contient» de la communication. Les approches de la «communication organisante» («communication as organizing» (Cooren, Taylor, Every 2006), " organisation-processus » analyse l'organisation «en train de se faire » vue comme un permanent processus de construction sociale.

Nicole d'Almeida et Yanita Andonova, dans leur analyse «historique » de l'évolution du champ de recherche en communication organisationnelle, considère que celui-ci «recouvre progressivement des recherches consacrées à l'analyse des processus communicationnels dans la configuration des organisations au sens large (entreprises, institutions publiques, collectivités territoriales, associations» (2006, p. 132). Malgré les diverses appellations de ce champ de recherche, celui-ci semble être le «sens commun »du mot organisation - l'équivalent du mot anglais « corporation ».

Les ACO (approches communicationnelles des organisations) innovent à ce niveau, en proposant une conception élargie de l'organisation qui «passe du statut d'objet d'étude à celui de catégorie conceptuelle» (Bouillon, Bourdin, Loneux, 2008, p. 6), désignant «un niveau intermédiaire de structuration sociale qui vise à penser les médiations entre local et global, individuel et collectif (idem). A l'instar de Christian Le Moënne, nous rejoignons une conception étendue de l'organisation, qui consiste à dépasser ses visions spatiales: "l'organisation n'est pas un état, mais un ensemble de processus perpétuels de rupture par rapport aux formes existantes et de recomposition des normes, de reconfiguration des figures, des agencements et équilibres divers qui apparaissent ainsi essentiellement provisoires et instables" (Le Moënne, 2003).

Nous approche vise à comprendre la dimension organisationnelle des pratiques sociales par la communication sans pour autant "réduire l'organisation à la communication" (Bouillon, 2009). La dimension organisationnelle est ainsi une dimension anthropologique constituée de normes et formes sociales héritées, de conventions non-choisies et nonvoulues mais ayant un statut symbolique de légitimes. Ces normes et formes héritées seraient à la base de ce que Simmel appelle "la maintenance de la société", en lui conférant une certaine "stabilité structurelle" (René Thom). C'est au niveau organisationnel que s'expriment de permanentes tensions entre tradition et innovation, entre prégnance et saillance (René Thom). Il ne s'agit pas pour nous de montrer l'existence d'une hiérarchie entre la tradition et l'innovation, mais au contraire de montrer que dans les pratiques sociales impliquant les nouvelles technologies se nouent, d'une façon indissociable, de formes pré-existantes et de formes émergentes.

\section{Quel rapport entre la notion d'organisation et la notion de pratiques?}

C'est à ce degré de conceptualisation qu'il est possible de faire un rapprochement entre la notion d'organisation et celle de pratique.

Les pratiques sociales sont pour Simmel des formes sociales situées et négociées en permanence et leur contexte est toujours organisationnel. Le réglage du volume sonore du téléphone portable, le geste de mettre un casque lorsqu'on écoute de la musique dans les transports en commun, ne sont pas de conduites anodines, mais elles fonctionnent pour 
les individus qui les réalisent comme des formes situées et négociées d'interaction. Ceci suppose l'existence d'un ordre observable et intelligible. "Les acteurs se comprennent dans la mesure où ils coordonnent leur action sur la base d'une construction conjointe de la visibilité de leur rencontre" (Loneux, 2008). Cette intercompréhension présuppose la prise en compte des normes anthropologiques et techniques. C'est sur cette "base" que les gens organisent leur vivre ensemble de façon à se rendre mutuellement intelligibles. Georg Simmel s'intéresse aux processus qui en dernier ressort se produisent dans les individus et qui conditionnent l'être-société (Gesellschafts-Sein) de ces individus. Il pose la question de la maintenance des formes sociales. Il nous semble que la question de la permanente émergence de nouvelles formes sociales et de nouvelles normes est toute aussi intéressante.

Notre argumentation se fait en faveur d'un retour des recherches en SIC sur la question organisationnelle - comme problématique heuristique dans les approches des TIC. Christian Le Moënne, remarque à maintes reprises (2004, 2008) que "la dimension organisationnelle est, non seulement négligée ou évitée, mais massivement sous-estimée dans l'appréhension comme dans la conceptualisation de phénomènes qui, pourtant, ne peuvent être compris que s'ils sont fortement référés à leurs contextes et situations d'émergence". Saisir la dimension organisationnelle des pratiques sociales revient donc à saisir les façons dans lesquelles les gens organisent leurs pratiques et leurs actions conjointes dans l'espace public et les modes de reconfiguration du rapport entre le monde vécu et le monde émergent.

Dans notre projet de recherche qui est en cours, nous nous intéressons aux pratiques des dispositifs nomades, notamment du téléphone portable, dans une approche comparative entre la France et la Roumanie. Nous interrogeons les tensions qui accompagnent l'“ancrage social" (Miège, 2007) des innovations techniques en s'appuyant sur l'exemple de la télévision sur le téléphone portable, les mutations qu'on peut observer lorsqu'une même innovation (la TV sur mobile) est introduite dans deux contextes éco-socio-culturels différents et de quelle façon se reconfigure le rapport entre les normes et formes sociales héritées et celles émergentes. Notre approche comparative ancrée dans deux contextes éco-socio-culturels différents (la Bretagne et la Transylvanie) prend tout son sens dans l'étude de ces tensions. Les normes techniques de même de que les normes anthropologiques évoluent en permanence, sans en être nécessairement conscients et elles influencent l'émergence de nouvelles formes sociales.

En effet, dans le contexte de la mondialisation et de l'espace économique et politique européen, les normes techniques de la TV mobile se propagent par le marché, par une volonté politique et un cadre législatif européen (Patrascu, 2010).

En même temps, les normes non-écrites de la "bonne" conduite dans l'espace public sont en continuelle évolution. En Roumanie, on a parlé après la chute du communisme d'une conduite "libérée". Or, cette libération du comportement, de la conduite en société et de l'apparition sur la scène publique se heurte souvent des normes de "bonne conduite" héritées. D'ailleurs, les comportements "déviants" sont vus comme tels justement quand il y a une transgression des règles, du cadre normatif, qu'il soit "naturel" ou conventionnel.

Garfinkel considère qu'afin de se rendre mutuellement intelligibles, les gens doivent faire preuve de ce qu'il appelle une "compétence organisationnelle". Cette compétence serait le fruit d'une validation intersubjective constante dans les interactions interhumaines et elle participerait à la régulation interne, au même titre que la confiance. Elle est selon Garfinkel (2007, Rééd.), une compétence à la fois sociale et pratique : sociale, car elle à un rôle déterminant dans la production de l'ordre, sa reconnaissance et son maintien et pratique, car elle suppose la maîtrise pratique des méthodes et procédés selon lesquels les 
diverses activités s'organisent. C'est sur le fond de cette compétence que les "incompétents" sont identifiés et le cas échéant informés et admonestés. Par exemple, lors de notre enquête, nous avons filmé deux situations quasi identiques à Rennes et à Timisoara : dans un milieu de transport en commun, un jeune écoute de la musique à un volume élevé sur son téléphone portable, sans utiliser de casque. Cette situation provoque des réactions de réprobation dans les deux terrains d'observation (des gestes, des regards, des discutions entre les autres passagers). Le jeune en cause a été rappelé à l'ordre uniquement dans le tramway de Timisoara. Les déviances, reconnues comme telles par une société, ont été dans ce cas, suivies par de tentatives plus ou moins subtiles de restauration du cours "normal" des choses. Finalement, la "subtilité" du rappel à l'ordre est à son tour le résultat d'une permanente confrontation avec ce que nous appelons "la dimension organisationnelle", constituée des normes et formes sociales et culturelles héritées.

Cela ne veut pas dire que ce que donne sens à une pratique ou simple activité, est sa cohésion, sa conformité absolue aux normes prédéfinies. Le sens se construit au cours de son élaboration située. Mais il reste que « en tant qu'elle se réalise progressivement, la société signifie toujours que les individus sont liés par des influences et des déterminations éprouvées réciproquement. Elle est par conséquent quelque chose de fonctionnel, quelque chose que les individus font et subissent à la fois" (Simmel, 1991). J. Freund, dans la préface de l'ouvrage de Simmel fait d'ailleurs la relation entre l'évolution des normes et l'évolution des formes. Il observe que : "L'action réciproque taille les formes de son devenir même, provoquant des ruptures et des failles (...). L'action réciproque transforme sans cesse la forme dans laquelle elle se produit, soit pour supprimer les relations, soit pour en créer de nouvelles, soit pour revivifier d'autres (p. 13-14)". Louis Quéré fait également le rapprochement entre la régulation des activités en commun par la norme et la régulation par la forme. "Les gens agissent sous une contrainte d'intelligibilité et de pertinence ; or leurs conduites ne sont intelligibles et reconnaissables comme appropriées que pour autant qu'elles manifestent, dans leur forme même, un certain nombre de traits, tels que la typicité, la standardisation, la répétitivité, la reproductivité, l'impersonnalité, la standardisation, la concordance, etc." (Quéré, 1988, p. 91).

\section{LA MEDIATION : UN CONCEPT FEDERATEUR}

Nous avons argumenté jusque-là en faveur d'un retour des approches des Tic aux problématiques liées à la dimension organisationnelle des pratiques sociales. Ce déplacement d'orientation est, à notre avis, susceptible de faire "travailler ensemble" les notions de pratique et d'organisation.

Nous poursuivrons cette réflexion en examinant comment les frontières entre ces notions et celle de médiation peuvent être dépassées à travers une approche communicationnelle de la dimension organisationnelle des pratiques sociales qui reposerait sur une prise en compte des médiations (techniques, sociales et symboliques) et des objets (vus comme médiateurs). La notion de médiation connaît une riche polysémie, tout comme les notions de pratique et d'organisation. Dans le dictionnaire Larousse (2010, dictionnaire en ligne), le mot "médiation" connaît quatre significations :

- Entremise, intervention destinée à amener un accord

- Fait de servir d'intermédiaire, en particulier dans la communication

- Procédure de règlement des conflits collectifs du travail dans laquelle intervient un médiateur.

- Mode de solution pacifique d'un conflit international, consistant à recourir à des médiateurs. 
Notre discipline s'est approprié depuis plusieurs années cette notion. Elle est ainsi devenue "à la fois une perspective de recherche et un modèle action" (Gellereau, 2006, p. 30). Bernard Lamizet (1992) va jusqu'à soutenir que "les sciences de l'information et de la communication sont des sciences de la médiation”. Employée au singulier ou au pluriel, la notion de médiation/médiations interroge le lien social, les dispositifs socio-techniques qu'on recouvre sous le nom de "média", des domaines professionnels bien identifiés (médiation culturelle, médiation familiale), un corps des métiers (médiateurs sociaux, juridiques) etc.

Nous ne nous intéresserons pas ici à la totalité des significations que cette notion connaît dans notre discipline. Ce qui nous intéresse ici est d'interroger les liens que ce concept entretient avec les notions de pratique et d'organisation. En poursuivant une orientation donnée par Jean Caune (1999), nous allons montrer que la notion de médiation reste riche du point de vue heuristique sur un axe horizontal, car elle permet de rendre compte des rapports entre les pratiques individuelles et les pratiques collectives, mais également sur un axe vertical, à travers la thématique de la mémoire et de la longue durée.

\section{La médiation "horizontale"}

Sur l'axe horizontal des relations interpersonnelles, la médiation se conçoit selon Lamizet dans la relation entre l'individuel et le collectif, entre le sujet singulier et la communauté à laquelle il appartient. La société ne peut exister que si "chacun de ses membres a conscience d'une relation entre sa propre existence et l'existence de la communauté" (Lamizet, 1999, p. 9). Ainsi, Lamizet, qui analyse notamment les médiations culturelles, considère que "la culture est l'ensemble des pratiques et des manifestations qui représentent symboliquement l'appartenance sociale de ceux qui les mettent en oeuvre" (Lamizet, 1999, p. 73).

Toujours dans cet axe de compréhension, la notion de médiation s'inscrit dans une logique de la teircité, de la présence d'un tiers. Pour Louis Quéré un des "tiers symbolisants", qui est à la base du "vivre-ensemble ", est l'institution: "La compréhension réciproque des sujets sociaux requiert la médiation symbolique d'un 'neutre'; elle fait appel au pôle de l'Institution, ce lieu où le lien social se noue. ». Ce qu'il appelle le tiers symbolisant, peut recouvrir une multiplicité de formes: "il est fait de l'articulation d'un ensemble d'éléments composites: des structures cognitives et des cadres normatifs, des repères de discrimination et des critères d'évaluation, des modes d'appréhension du temps et des dispositions vis-à-vis du changement, des règles de choix et des propositions définissant des façons de procéder (technologies), des modes de représentation et des schémas d'action; à un autre niveau, des jeux de rôles et des catégories de la pratique, des affirmations considérées comme vraies et des normes tenues pour justes, des croyances et des figurations" (1982, p. 83) C'est dans ce sens de la teircité que nous pouvons parler de multiples médiations (techniques, sociales, culturelles, esthétiques, etc.) dans lesquelles les tiers-médiateurs sont les dispositifs techniques, les normes et codes anthropologiques, les formes objectales, etc.

Sur l'axe horizontal des relations interpersonnelles on peut donc dire que, dans une perspective communicationnelle, la notion de médiation permet de rendre compte de l'inscription des pratiques individuelles dans les pratiques collectives. Les multiples formes de médiations (techniques, sociales, symboliques) rendent possible le vivre-ensemble.

\section{Médiation sur l'axe vertical du rapport au temps}

Nous soulignons ici deux significations que la notion de médiation recouvre sur un axe vertical. Dans un premier temps, nous interrogeons les dispositifs techniques vus comme 
dispositifs de mémoire puis vus comme dispositifs de médiation entre l'homme et la nature.

\section{Les dispositifs sociotechniques — dispositifs de mémoire?}

Le concept de médiation interroge dans un axe vertical, du rapport au temps, la notion de mémoire. Dans ce sens, nous soutenons que les objets techniques sont des dispositifs de médiation en tant que dispositifs de mémoire. La mémoire est ainsi conçue comme une médiation.

Simmel posant la question de la maintenance de la société, souligne l'un des facteurs qui rend la vie en société possible : le sol, le sentiment d'appartenir à un territoire. Cependant, ce facteur n'est pas suffisant; pour Simmel ce qui permet la permanence du collectif s'est avant tout "liaison physiologique des générations" (Simmel, 1896-1897, p. 77). En effet, les générations se succèdent continuellement, "la sortie des éléments anciens et l'entrée de nouveaux s'opèrent si progressivement que le groupe fait l'effet d'un être unique" (p. 77). "C'est ce renouvellement lent et progressif du groupe qui en fait l'immortalité" (p. 78).

Sans vouloir faire des rapprochements artificiels entre concepts et auteurs, cette liaison entre générations est interrogée un penseur de la technique: Gilbert Simondon. Cette question de la "liaison physiologique" entre les générations se retrouve débattue par Simondon à travers le concept de pré-individuel. La réflexion de Simondon porte sur l'individu et le collectif, mais surtout sur le processus d'individuation, c'est-à-dire "le passage du bagage psychosomatique générique de l'animal humain à la configuration d'une singularité unique". Pour Simondon, la vie sociale, donc la vie en collectivité, ne s'oppose pas au processus d'individuation. Dans la vie en groupe, la singularité se perfectionne et fait des efforts pour mieux s'exprimer. La vie sociale, est dans la conception de Simondon, la conjoncture d'une ultérieure et plus complexe individuation: " $L a$ participation [au social], pour l'individu, est le fait d'être élément dans une individuation plus vaste par l'intermédiaire de la charge de réalité préindividuelle que l'individu contient, c'est-à-dire grâce aux potentiels qu'il recèle»(1964, p. 11) L'individu devient partie du groupe par la réalité préindividuelle qu'il porte en lui et qui, réunie à celle d'autres individus, forme une unité collective. La relation au monde et au collectif est une dimension de l'individuation à laquelle participe l'individu à partir de la réalité préindividuelle qui s' "individue" progressivement (Stiegler, 2003) : "l'individu a conservé avec lui du pré-individuel, et tous les individus ensemble ont ainsi une sorte de fond non structuré à partir duquel une nouvelle individuation peut se produire». (Simondon, 1989, p. 193) Simondon considère que les "êtres" sont rattachés les uns aux autres dans le collectif, en tant que sujets c'est-à-dire en tant "qu'êtres qui contiennent du pré-individuel" (Simondon, 1989, p. 205). L'élément préindividuel présent dans chaque sujet se situe donc au fondement du vivre-ensemble.

Selon Bernard Stiegler, fin lecteur de Simondon, tout individu se développe à partir de trois mémoires : mémoire génétique (ou spécifique), mémoire épigénétique (ou nerveuse) et enfin, mémoire épiphylogénétique (ou techno-logique). Stiegler définit son principe d'épiphylogenèse : "L'épiphylogenèse désigne l'apparition d'un nouveau rapport entre l'organisme et son milieu, nouveau rapport qui est aussi un nouvel état de la matière : si l'individu est une matière organique et donc organisée, son rapport au milieu (à la matière en général, organique et inorganique) est médiatisé par cette matière organisée quoique inorganique qu'est l'organon, l'outil avec son rôle instructeur (son rôle d'instrument) »(2003)

Dans une autre perspective, Bernard Lamizet considère que la question de la mémoire individuelle et collective est en lien direct avec celle de la culture. En effet, la mémoire individuelle "n'est finalement que l'appropriation individuelle des représentations liées au passé. La mémoire met en ouvre tout un ensemble de processus psychologiques par 
lesquels le sujet individuellement, se projette dans le temps en inscrivant dans une continuité unifiée tous les événements, toutes les situations dans lesquels il est impliqué" (Lamizet, 1992, p. 140). Dans ce sens, la mémoire est une expérience individuelle. La mémoire collective peut être envisagée comme un médiateur "la mémoire est faite d'images, de représentations que nous avons en commun avec les autres». (Lamizet, 1992, p. 140).

La dimension organisationnelle définie dans la partie ultérieure comme une dimension anthropologique faite des permanents processus de confrontation entre héritage et innovation pose comme primordiale la question de la mémoire individuelle et collective. Car les formes et les normes traditionnelles sont reconnues comme telles par un travail de mémoire. La mémoire (voire les différents types de mémoire) participe dans ce sens à la constitution de la dimension organisationnelle.

\section{Les dispositifs socio-techniques - dispositifs de médiation entre l'homme et la nature}

La conception des objets techniques de Gilbert Simondon intéresse depuis quelques années les chercheurs en SIC. Dans notre tentative de faire travailler ensemble les notions de pratique, d'organisation et de médiation, la pensée de Simondon est d'une grande richesse heuristique. Nous retrouvons chez lui les notions de médiation et de médiateurs. Pour lui, les objets sont des médiateurs entre l'humain et le naturel et les humains sont de créateurs des médiations: "Le travail est ce par quoi l'être humain est médiateur entre la nature et l'humanité comme espèce [...] par l'activité technique, l'homme crée des médiations" (Simondon, 1969, p. 245). C'est par la concrétisation, exprimée à travers un permanent processus conjoint de création/ négociation et remise en forme aussi bien de l'objet technique que de l'environnement, que les objets techniques peuvent être considérés comme des médiateurs entre le naturel et l'humain. Et l'activité technique est celle qui "rattache l'homme à la nature selon un lien beaucoup plus riche et mieux défini que celui de la réaction spécifique de travail collectif" (idem).

\section{CONCLUSION}

La spécificité des SIC consiste dans le point de vue - communicationnel, qu'elles portent sur les dispositifs socio-techniques de communication. Une posture qui exige "pour les chercheurs en information-communication, " de ne pas "assimiler les objets techniques de la communication aux autres" (Y. Jeanneret, 2009). C'est sur ce point que les travaux en SIC se distinguent: la conceptualisation de l'information - communication (Miège, 2004) et la vision "des machines à communiquer" vues comme médias. "L'utilisation d'un livre, d'un micro-ordinateur ou d'un téléphone mobile n'est pas la combinaison de logiques sociales et de propriétés techniques. Ces objets sont des médias, c'est-à-dire des objets qui ne font pas que s'associer à du social, mais qui en produisent” (Y. Jeanneret, 2009).

Les différentes appréhensions des trois notions: pratique, organisation et médiation effleurent souvent les problématiques communicationnelles susceptibles justement à notre avis de faire "travailler ensemble" ces notions. Entre ces trois notions, notion du sens commun, transformée en concept, la notion de médiation par les problématiques communicationnelles qu'elle interroge, permet de "penser ensemble" les notions de pratique et d'organisation aussi bien dans un axe horizontal en permettant de saisir la dimension organisationnelle des pratiques individuelles et collectives, mais également dans un axe vertical, celui qui oriente l'appréhension des médiations inscrites dans une temporalité longue. Le concept de médiation nous permet de dépasser la dialectique entre la dimension matérielle et sémiotique des objets de médiation et la dimension organisationnelle, anthropologique des façons de faire. 


\section{REFERENCES BIBLIOGRAPHIQUES}

Bernard, Françoise (2000), "Le lien communicationnel en organisation", Sciences de la Société, 50/51, 2000, p. 25-45.

Bouillon, Jean-Luc (2009) "Comprendre l'organisation par la communication... sans réduire l'organisation à la communication”. 77e Congrès de l'ACFAS, Ottawa, mai 2009.

Bouillon, Jean-Luc, Bourdin, Sylvie, Loneux Catherine (2008), “Approches communicationnelles des organisations : interroger l'organisation par la communication, éléments de contextualisation”. Sciences de la Société, n 74 , p. 3-9.

Caune, Jean (1999), "La médiation culturelle : une construction du lien social". Les enjeux de l'information et de la communication (en ligne)

http://w3.u-grenoble3.fr/les_enjeux/2000/Caune/home.html, page consultée le 01 mai 2010.

F. Cooren, J. R. Taylor \& E. J. Van Every (Eds.) (2006), Communication as Organizing : Empirical and Theoretical Explorations in the Dynamic of Text and Conversation. London: Laurence Earlbaum.

D'Almeida Nicole, et Andonova Yanita (2006) "La communication des organisations", in Olivesi (S.) (dir.), Sciences de l'Information et de la Communication. Objets, savoirs, discipline, Grenoble : PUG, p. 129-143.

Delcambre Pierre (2000), Communications organisationnelles. Objets, pratiques, dispositifs, Rennes : PUR.

Garfinkel, Harold (2007), Recherches en ethnométhodologie, Paris : PUF, 2007 (Réédition)

Jeanneret, Yves (2009), "La relation entre médiation et usage dans les recherches en information- communication en France". RECIIS - Electronic. Journal of Communication, Information and Innovation in Health. Rio de Janeiro, v.3, n.3.

Jouët, Josiane (1993), "Usages et pratiques des nouveaux outils de communication”. In L. Sfez (Ed.), Dictionnaire critique de la communication. Paris : PUF.

Lamizet, Bernard (1992), Les lieux de la communication. Liège, Mardaga.

Lamizet, Bernard (1999), La médiation culturelle, Paris, Editions L'Harmattan.

Le Moënne, Christian (2008), "La "question organisationnelle" pour les sciences de l'information et de la communication". Les Cahiers de la SFSIC, № 3, juin 2008.

Le Moënne, Christian (dir.) (2004), "Systèmes d'information organisationnels ?”. Sciences de la société, 61, Toulouse : PUM.

Le Moënne, Christian (2003), "Questions et hypothèses sur les approches constructivistes et les recherches en communications organisationnelles". Dans Centre d'étude et de recherche sur l'information et la communication (Ed). La Place du constructivisme pour l'étude des communications. Actes du colloque de Béziers (pp. 9-44), Montpellier: ED C2M2.

Loneux, Catherine (2007), L'éthique entrepreneuriale et managériale comme dispositif communicationnel: analyse des enjeux et pratiques - problématisations. Mémoire d'Habilitation à Diriger des Recherches : Aix Marseille 1.

Miège, Bernard (2007), La société conquise par la communication. Tome III. Les Tic entre innovation technique et ancrage social, PUG. 
Miège, Bernard. "Questionnement de la sociologie des usages comme voie privilégiée de l'approche des TIC". Article disponible en ligne, www.u-grenoble3.fr/gresec/pagespublic/documents/miege_AISLF.doc.

Miège, Bernard (2004), L'information-communication, objet de connaissance, Bruxelles/Paris : de Boeck/ INA.

Patrascu, Marcela (2010), "Insertion de nouveaux produits : permanente confrontation avec les normes et formes culturelles héritées". in Actes du colloque Culture et identités dans les communications marketing, 78e Congrès de l'ACFAS, Université de Montréal, 10-14 mai 2010. Actes disponibles en ligne,

http://www.crpcm.uqam.ca/Pages/comm_mark_pub.aspx.

Proulx, Serge (2005), "Penser les usages des technologies de l'information et de la communication aujourd'hui : enjeux - modèles - tendance". In Lise Vieira et Nathalie Pinède, éds, Enjeux et usages des TIC : aspects sociaux et culturels, Tome 1, Bordeaux : Presses universitaires de Bordeaux, p. 7-20.

Quéré, Louis (1982), Des miroirs équivoques, Paris : Aubier.

Quéré, Louis (1988), “Sociabilité et interactions sociales”. Réseaux, Volume 6, N²9, p. 75 91.

Quéré, Louis (1990), “Agir dans l'espace public”. In Pharo, P. et Quéré L. Les formes de l'action. Paris : EHESS, (Raisons Pratiques I).

Sacks, Harvey (1993), "La description sociologique". Cahiers de recherche ethnométhodologique, 1, Paris :Ed. Paris VIII, 1993, p. 7-23.

Simmel, Georg (1992), Sociologie et Epistémologie, Paris : PUF (réed).

Simmel, Georg (1896-1897), “Comment les formes sociales se maintiennent”, Année Sociologique I, p. 71-109.

Stiegler, Bernard (2003), "Temps et individuation technique, psychique, et collective dans l'œuvre de Simondon". Article mis en ligne le 23 octobre 2003. Disponible en ligne : http://multitudes.samizdat.net/Temps-et-individuation-technique.html\#nb14\#nb14 [dernière consultation : le 20/11/09].

Simondon, Gilbert (1964), L'individu et sa genèse physico-biologique, Paris: PUF, col. Epiméthée.

Simondon, Gilbert (1989), L’individuation psychique et collective. Paris : Aubier.

Simondon, Gilbert (1969), Du mode d'existence des objets techniques. Paris: Aubier. 